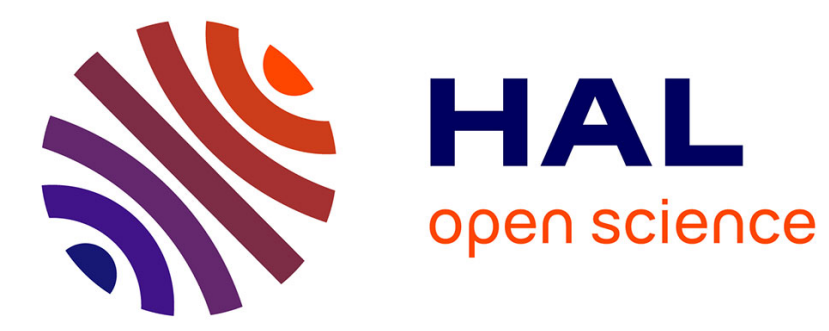

\title{
La troisième personne du pluriel (indicatif présent) autour de Metz : une révolution paradigmatique
}

Marc Duval

\section{To cite this version:}

Marc Duval. La troisième personne du pluriel (indicatif présent) autour de Metz : une révolution paradigmatique. Langages, 2019, $\mathrm{N}^{\circ} 215$ (3), pp.57. 10.3917/lang.215.0057 . hal-02372567

\section{HAL Id: hal-02372567 \\ https://hal.sorbonne-universite.fr/hal-02372567}

Submitted on 20 Nov 2019

HAL is a multi-disciplinary open access archive for the deposit and dissemination of scientific research documents, whether they are published or not. The documents may come from teaching and research institutions in France or abroad, or from public or private research centers.
L'archive ouverte pluridisciplinaire HAL, est destinée au dépôt et à la diffusion de documents scientifiques de niveau recherche, publiés ou non, émanant des établissements d'enseignement et de recherche français ou étrangers, des laboratoires publics ou privés. 


\title{
La troisième personne du pluriel (indicatif présent) autour de Metz : une révolution paradigmatique
}

\section{The third person plural (indicative present) around Metz : a paradigmatic revolution}

Marc Duval

Sorbonne Université

\section{Résumé}

La troisième personne du pluriel de l'indicatif présent fait l'objet d'un traitement particulier dans les variétés galloromanes de la région de Metz : contrairement aux autres dialectes lorrains, les dialectes de cette zone ne marquent pas P6 par un suffixe vocalique, ce qui a pour conséquence que la troisième personne du pluriel est soit homonyme des personnes du singulier, soit marquée par une base spécifique du pluriel. Cette situation étant précisément celle que connaît le français moderne (cf. ils aim-[ent] vs nous aim-ons, elles finiss-[ent] vs nous finiss-ons), nous supposons une influence du standard sur les variétés dialectales autour de Metz, où cette ville joue depuis longtemps un rôle de pivot dans la propagation à la fois du standard et du dialecte.

Mots-clés

troisième personne du pluriel, indicatif présent, traits de temps et nombre, dialectes lorrains romans, Metz

\begin{abstract}
The third person singular indicative present is given a specific treatment in Gallo-Romance varieties in the region of Metz : unlike other Lorraine dialects, dialects of this area do not display a vocalic suffix for $\mathrm{P} 6$, which implies either that the third person plural is homonymous with the singular, or that it is marked by a particular plural base. As this situation is precisely that known in Modern French (see ils aim-[ent] vs nous aim-ons, elles finiss-[ent] vs nous finiss-ons), it will be argued that the Standard has influenced dialectal varieties around Metz, a region in which this city has for a long time played a pivotal role in conveying both Standard and dialectal forms.
\end{abstract}

Keywords

third person plural, indicative present, tense and number features, Romance dialects of Lorraine, Metz 


\section{INTRODUCTION ${ }^{1}$}

Les données des variétés discutées, notamment celles tirées des atlas dialectaux, seront présentées sous forme de paradigmes simplifiés où les formes verbales sont remplacées par des lettres de l'alphabet, chaque lettre étant attribuée à une forme flexionnelle distincte des autres. Le paradigme des verbes à une seule base en français moderne standard sera donc représenté par la séquence aaadea à l'indicatif présent au code oral, la répétition de la lettre $a$ permettant de signaler l'homonymie des formes verbales associées à $\mathrm{P} 1, \mathrm{P} 2, \mathrm{P} 3$ et $\mathrm{P} 6$ :

Tableau 1 : Paradigme verbal du français standard
\begin{tabular}{|l|l|l|l|}
\hline Code écrit & a & Code oral \\
\hline (j') aim-e & bm- $]$ & a \\
\hline (tu) aim-es & b & {$[\varepsilon m-\varnothing]$} & a \\
\hline (il) aim-e & $\mathrm{a}$ & {$[\varepsilon m-\varnothing]$} & $\mathrm{a}$ \\
\hline (nous) aim-ons & $\mathrm{d}$ & {$[\varepsilon \mathrm{m}-\tilde{o}]$} & $\mathrm{d}$ \\
\hline (vous) aim-ez & $\mathrm{e}$ & {$[\varepsilon \mathrm{m}-\mathrm{e}]$} & $\mathrm{e}$ \\
\hline (ils) aim-ent & $\mathrm{f}$ & {$[\varepsilon \mathrm{m}-\varnothing]$} & $\mathrm{a}$ \\
\hline
\end{tabular}

Comme on le sait, cette situation de forte homonymie des formes verbales fléchies est une caractéristique du français qui le distingue des autres langues romanes standard. Naturellement, les variétés dialectales galloromanes, en domaine d'oïl en premier lieu, connaissent également ce type d'homonymies, mais selon des structurations paradigmatiques spécifiques. Nous faisons l'hypothèse que les paradigmes flexionnels constituent un paramètre essentiel et prometteur non seulement dans la description de la segmentation dialectale, mais aussi dans la compréhension de la dynamique et de l'interaction des différentes variétés en présence.

On dispose, pour la Meurthe-et-Moselle et les Vosges, de données importantes sur les paradigmes verbaux à la fin du XIX ${ }^{\mathrm{e}}$ siècle. Celles-ci ont été réunies par L. Adam (1881), qui notait à juste titre que les parlers de Lorraine tendent d'une manière générale à opposer une forme pour le singulier à une forme pour le pluriel. Adam parle de « conjugaison biflexionnelle » :

Tandis que la langue anglaise a débarassé $[s i c]$ ses verbes de la presque totalité de leurs flexions devenues absolument inutiles, les personnes étant désignées par des pronoms, le français ne s'est point affranchi du joug des désinences de la conjugaison

\footnotetext{
${ }^{1}$ Nous tenons à remercier particulièrement messieurs J.-P. Chambon pour ses conseils utiles lors d'une « consultation » improvisée par téléphone, M.-D. Glessgen pour nous avoir suggéré l'existence de deux grands axes de communication en Lorraine qui ne se confondent pas depuis le Moyen Âge, J.-B. Picard pour nous avoir communiqué des photos des mémoires inédits de l'enquête de L. Adam, ainsi que nos relecteurs. Les données de 1'ALLR suivent la notation Rousselot-Straka, celles de R. Brod, F. Dosdat, C. This et L. Zéliqzon (1889) empruntent le système de Böhmer et de la Zeitschrift für romanische Philologie; dans ses travaux ultérieurs, L. Zéliqzon utilisera le système de la Société de Littérature Wallonne. Certains signes n'ont pu être repris de la police TimesUnicodeCD, à l'instar des semi-nasales de l'ALLR (non trancrites) ou du schwa du système Böhmer (remplacé ici par le schwa de l'API).
} 
latine. Quelques-uns de nos patois y sont également demeurés assujettis, mais la plupart se sont ingéniés á (sic) diminuer les difficultés inhérentes á la conjugaison flexionnelle, en réduisant à deux le nombre des flexions, dans la majorité des Temps. Une flexion pour le singulier, une flexion pour le pluriel, telle a été en Lorraine, comme en Bourgogne, la solution très-rationnelle du problème. Exemple : Imparfait : dje prakè, te prakè, é prakè, dje prakonne, vos prakonne, è prakonne (Ban-surMeurthe). (Adam, 1881 : xxxix)

Le paradigme verbal idéal en Lorraine serait donc de type aaaddd. En réalité, ce paradigme, largement attesté par ailleurs, est absent de l'indicatif présent. On peut considérer que c'est la corrélation du système du présent avec le système de l'impératif, à l'instar du français moderne, qui contraint l'érosion des formes du présent (Duval 2009) : l'impératif requiert en effet une opposition minimale entre les trois personnes P2 (aim-[e]), P4 (aim-ons) et P5 (aim-ez) que l'on retrouve au présent de l'indicatif. Cela ne dit rien toutefois de la troisième personne du pluriel, qui est, sans danger pour le système, libre de s'aligner par exemple avec les personnes du singulier comme en français moderne.

Or, les dialectes lorrains présentent normalement un système où la troisième personne du pluriel est toujours distincte des personnes du singulier, soit des systèmes aaaddd, aaaded, et, plus rarement, aaadef.

\section{Verbes A bASE UNIQUE}

C'est dans un tel contexte que les données des dialectes de la région de Metz prennent un poids particulier, puisqu'ils ont régulièrement, pour les verbes à une base, un système de type aaadea. On comparera, dans le tableau suivant, le paradigme aaaded de «mettre (= bouter)» à Labaroche dans les Vosges alsaciennes, le paradigme aaaded ou aaadef du verbe « aimer » à Château-Salins, commune de Moselle (Brod, 1912), enfin le paradigme aaadea du verbe « craindre (= douter) » pour les dialectes de Moselle parlés autour de Metz (Zéliqzon, 1922 : xvi) :

\begin{tabular}{|c|c|c|c|c|c|c|}
\hline \multirow[b]{2}{*}{ P1 } & \multicolumn{2}{|c|}{ Labaroche } & \multicolumn{2}{|c|}{ Château-Salins } & \multicolumn{2}{|c|}{ MPIN (Metz) $)^{2}$} \\
\hline & [bot] & a & [ēm] & a & [dǫt] & a \\
\hline $\mathrm{P} 2$ & [bot] & $\mathrm{a}$ & [ēm] & $a$ & [dęt] & $\mathrm{a}$ \\
\hline P3 & [bot] & a & [ēm] & a & [dot] & a \\
\hline $\mathrm{P} 4$ & [boto] & $\mathrm{d}$ & [ēmõ], [ēmã] & $\mathrm{d}$ & [dọtã] & $\mathrm{d}$ \\
\hline P5 & [botō] & $\mathrm{e}$ & [ēmō] & $\mathrm{e}$ & [dǫtōe] & $\mathrm{e}$ \\
\hline P6 & [boto] & d & [ēmõ] & $d / f$ & [dǫt] & a \\
\hline
\end{tabular}

Si l'on considère la répartition dans l'espace global de la Lorraine sur la base de l'ALLR, on constate l'existence d'une zone continue autour de Metz, formée par

\footnotetext{
${ }^{2}$ Les lettres MPIN correspondent aux dialectes mosellans respectivement «messin, du Pays-Haut, de l'Isle, et de la Nied », par opposition, avec ceux du Saunois (S) et des Vosges du nord (V).
} 
seize localités (points $36,38-46$, et 56-60), ainsi que trois autres points d'enquête entre Verdun et Bar-le-Duc en Meuse. Voir la carte suivante ${ }^{3}$ :

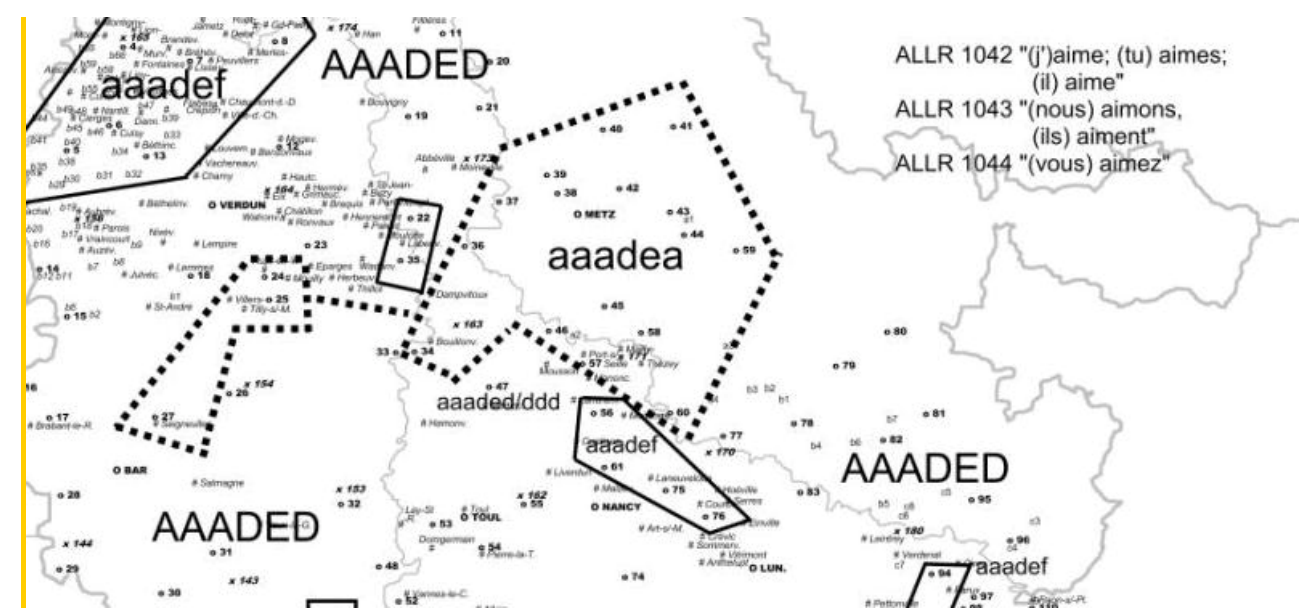

Figure 1 : Synthèse des cartes 1042, 1043, 1044 de l'ALLR

On peut voir sur cette carte les trois systèmes principaux qui se partagent l'espace dialectal lorrain :

- (i) (aaa)ded: structure dominante, uniformément répartie, et manifestement novatrice en raison de l'homonymie de P4 et de P6 ;

- (ii) (aaa)def: structure dispersée dans l'espace, probablement plus archaïque, en raison de la différenciation de $\mathrm{P} 4$ et de P6, sans confusion toutefois entre singulier et pluriel ;

- (iii) (aaa)dea: structure liée à une aire proche d'un centre urbain (Metz), qui est novatrice en raison de l'homonymie P1-P3 et P6, à l'instar du français.

Ceci pour les verbes à base unique. Pour d'autres verbes du premier groupe, on pourra consulter les cartes «commencent» (ALF 311), «crèvent » (ALF 353), « brillent » (ALLR 3), « jettent » (ALLR 49), « pondent» (ALLR 317), « frappent (du bec)» (ALLR 328), « carillonnent» (ALLR 1273), qui dessinent une zone similaire.

\section{VeRBES A PLUS D'UNE BASE}

\subsection{Présentation}

En français moderne standard, il existe deux grands systèmes de répartition des bases verbales en fonction de l'alignement de la troisième personne du pluriel au

\footnotetext{
${ }^{3}$ Nos fonds de cartes reportent également les points d'enquête de L. Adam, de l'ALF, d'O. Bloch (1917) et de J. Babin (1954). Sauf indication contraire, seules les données de l'ALLR sont reportées. Nous ne discuterons pas les localités des Vosges méridionales, de type franc-comtois.
} 
présent de l'indicatif. Soit cette P6 s'aligne sur les personnes du singulier, avec l'usage de la base "courte " sans marque personnelle à l'oral (de même pour le présent du subjonctif), ce qui donne une distribution en « $\mathrm{L} »$ de la base longue dans le tableau suivant :

Tableau 3 : Verbes en « L » (appeler, croire, voir, mourir, etc.)
\begin{tabular}{|l|l|l|l|}
\hline Appeler & imparfait & présent & subjonctif \\
\hline j'-il & appel- & appell- & appell- \\
\hline ils & appel- & appell- & appell- \\
\hline ns/vs & appel- & appel- & appel- \\
\hline
\end{tabular}

Soit elle prend la base longue, et s'aligne ainsi sur celle des autres personnes du pluriel, et de l'ensemble des personnes de l'imparfait et du subjonctif présent, ce qui forme une distribution en « $\mathrm{U} »$ :

Tableau 4: Verbes en « U» (finir, construire, vendre, battre, mettre, suivre, vivre, dormir, etc.)

\begin{tabular}{|l|l|l|l|}
\hline finir & imparfait & présent & subjonctif \\
\hline j'-il & finiss- & fini- & finiss- \\
\hline ils & finiss- & finiss- & finiss- \\
\hline ns/vs & finiss- & finiss- & finiss- \\
\hline
\end{tabular}

\begin{tabular}{|l|l|l|l|}
\hline vendre & imparfait & présent & subjonctif \\
\hline j'-il & [vãd]- & [vã]- & [vãd]- \\
\hline ils & [vãd]- & [vãd]- & [vãd]- \\
\hline ns/vs & [vãd]- & [vãd]- & [vãd]- \\
\hline
\end{tabular}

Le paradigme des formes finies est alors respectivement aaadea pour les verbes en « $\mathrm{L}$ » et aaadef pour ceux en « $\mathrm{U}$ ».

Pour la Lorraine, l'ALLR permet de récolter des données pour deux sous-types de ces verbes en $\left\langle\mathrm{U} »^{4}\right.$ : vendre ainsi que le paradigme typique d'un verbe du deuxième groupe.

\subsection{Le type « vendre »}

Pour vendre, on retrouve un système similaire - sans être identique - au français moderne standard : en effet, dans certains dialectes, la troisième personne du pluriel n'a pas de suffixe flexionnel propre, mais se distingue des autres personnes du pluriel par une base à finale consonantique au lieu d'avoir un suffixe personnel clairement identifiable, comme c'est le cas général en Lorraine. Voici les formes en question :

\footnotetext{
${ }^{4}$ Malheureusement, les données sont insuffisantes pour rendre compte des verbes en « $\mathrm{L} »$, y compris ceux du premier groupe.
} 


\begin{tabular}{|c|c|c|c|c|c|c|}
\hline & \multicolumn{2}{|c|}{$\begin{array}{l}\text { Metz (36, 38-46, } \\
56-60)\end{array}$} & \multicolumn{2}{|l|}{24,25} & \multicolumn{2}{|l|}{27} \\
\hline $\mathrm{P} 1$ & vã $\left({ }^{\eta}\right)$ & $a$ & vò/vó & $a$ & và & a \\
\hline $\mathrm{P} 2$ & vã( $\left({ }^{\mathrm{\eta}}\right)$ & $a$ & vò/vó & $a$ & và & $a$ \\
\hline P3 & vã( $\left({ }^{7}\right)$ & $a$ & vò/vó & a & và & $a$ \\
\hline P4 & vãdã & $d$ & vòdã & $d$ & vàdõ & $d$ \\
\hline P5 & vãdói & e & vòdé & $\mathrm{e}$ & vàdố & e \\
\hline P6 & vã̃t & $f$ & vòt & $f$ & vàt & $f$ \\
\hline
\end{tabular}

Sur la carte suivante, on constatera que la zone correspondante, marquée « def* », coïncide avec celle déjà indiquée en 2.1 :

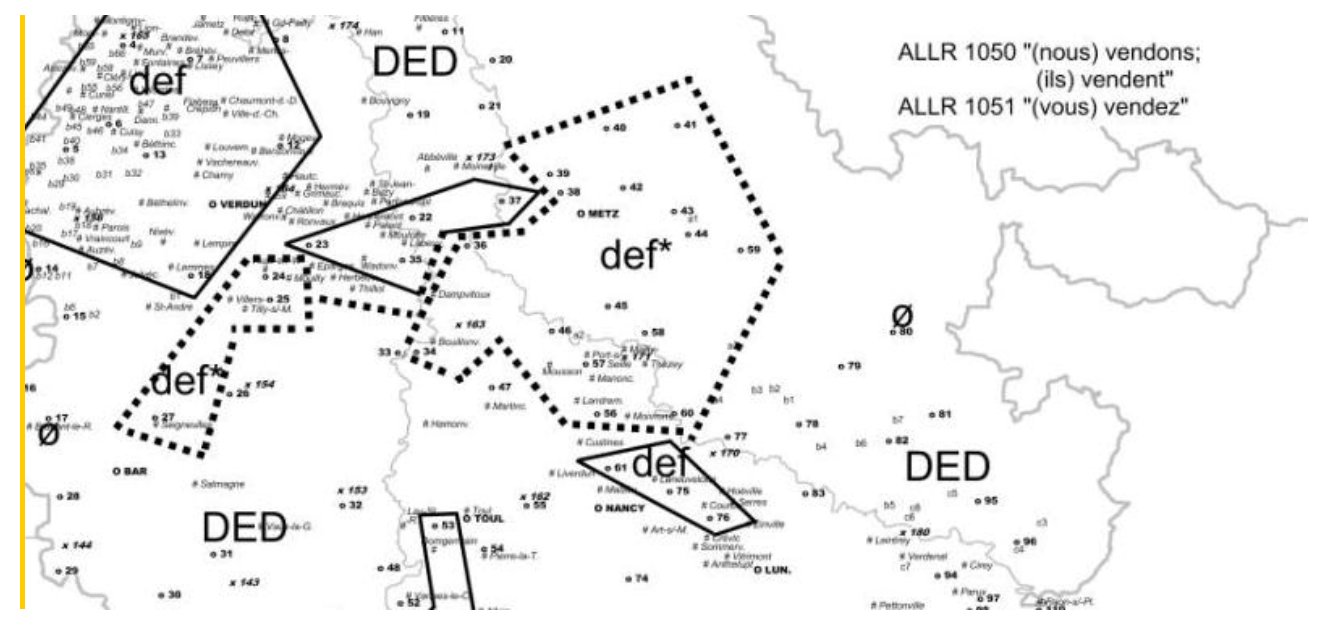

Figure 2 : Synthèse des cartes 1050, 1051 de l'ALLR

En réalité, le système n'est pas tout à fait homologue du français, puisqu'il y a trois bases phonétiquement différentes : $v \mathrm{~V}, v \mathrm{~V} d$-, et $v \mathrm{~V} t$, là où l'équivalent français n'a que deux bases (ven $[d]$ - et vend-). Toutefois, on peut faire intervenir une règle phonologique de dévoisement de la finale pour expliquer la présence d'une base $v \mathrm{~V} t$ manifestement issue de la base $v \mathrm{~V} d^{5}$.

\subsection{Le type « choisir, finir »}

La carte schématique de l'opposition P4/P6 pour le verbe choisir, en notant seulement les finales, permet de faire ressortir encore la singularité de notre zone :

\footnotetext{
${ }^{5}$ La désonorisation des consonnes finales est effectivement attestée dans les parlers lorrains (ainsi que parlers wallons et français régionaux), sans être toutefois exempte d'exceptions. Voir M. A. Borodina (1966 : 127-129) pour une discussion à partir des cartes de l'ALF.
} 


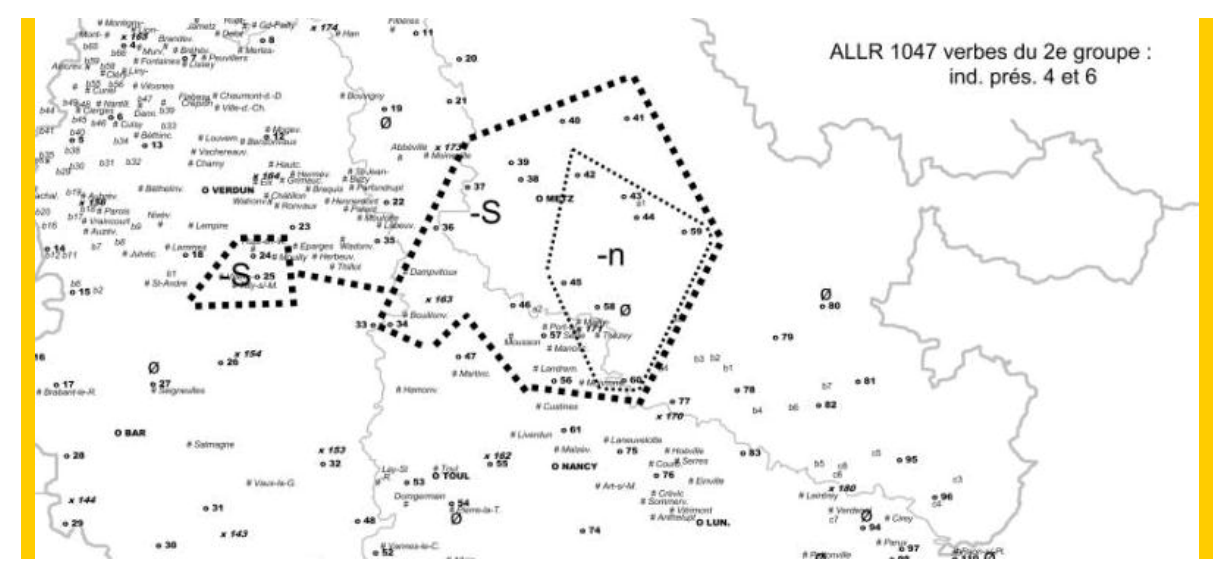

Figure 3 : Carte 1047 de l'ALLR

Dans les zones en pointillés, choisir à la troisième personne du pluriel de l'indicatif présent a comme vendre une finale consonantique (type choisiss- $\varnothing$, ou choisin). De structure aaadef, il est en cela identique au système français.

Le caractère exceptionnel de cette zone frappe dans le cadre plus général des parlers d'oïl. La carte 574 de l'ALF (« (ceux qui) finissent»), quoiqu'excluant la Moselle qui relevait alors de l'Empire allemand, montre aussi des finales consonantiques dans des parlers de Meurthe-et-Moselle à proximité de la région messine (on inclut la variante [ho:t] de halten pour le point 170).

Notre zone, quoique bien séparée du reste du domaine, s'aligne donc sur le système général de la majorité des parlers d'oïl de l'ouest, excepté les parlers de la Mayenne et de la Sarthe, qui marquent, comme l'essentiel des parlers lorrains, la P6 par une finale vocalique. 


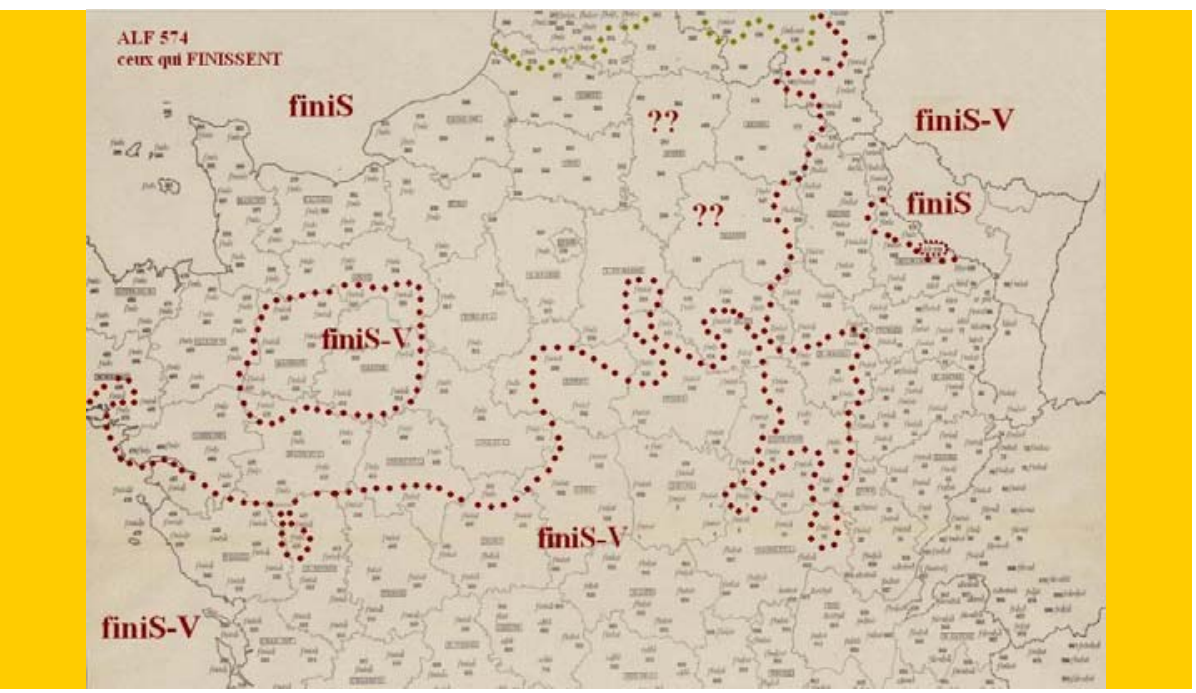

Figure 4 : Carte 574 de l'ALF : « (ceux qui) finissent »

Étant donné que la zone délimitée est à la fois compacte et tout à fait exceptionnelle du point de vue des parlers lorrains, il est utile de la situer aussi précisément que possible dans l'espace et dans le temps (§ 3). Les formes marquées par un $-n$ final (type choisin), notées par C. This (1887), L. Zéliqzon (1889) ou F. Dosdat (1909), sont un développement spécifique que les données modernes situent à l'Ouest de Metz. C. This fait l'hypothèse, reprise par W. Meyer-Lübke (1895: 199), qu'il s'agit d'une rétention du $n$ du suffixe latin de P6 -ent. Cette proposition pose un certain nombre de problèmes du point de vue de la phonétique diachronique, de la consistance du paradigme (seules certaines classes de verbes, excluant les verbes du premier groupe, sont marqués ainsi, et de la variation importante qu'on y trouve (p.ex. $d \bar{x} n$ et $d \bar{x} f$ pour « doivent»), signe probable d'un bouleversement relativement récent qui relève des bases verbales, à l'instar de la variation observée pour le suffixe -iss (cf. fleurent pour «fleurissent» ou mentissez pour « mentez $»)$. Nous reportons cette discussion à un autre travail.

Quoi qu'il en soit, la zone où P6 se termine en - $n$ pour une partie des verbes est d'une manière absolument régulière caractérisée par l'absence d'un suffixe personnel vocalique, et s'intègre en cela à l'ensemble ci-dessus délimité.

\section{SitUATION GEOGRAPHIQUE ET HISTORIQUE}

Les données rassemblées de diverses sources qui indiquent des finales consonantiques pour P6 permettent d'identifier les localités suivantes : 


\begin{tabular}{|c|c|}
\hline - ALLR : & $\begin{array}{l}\text { Moselle : (38) Woippy, (39) Norroy-le-Veneur, } \\
\text { (40) Trémery, (41) St-Bernard, (42) Failly, (43) } \\
\text { Silly-sur-Nied, (44) Maizeroy, (45) Goin, (58) } \\
\text { Secourt, (59) Hémilly, (60) Manhoué ; } \\
\text { Meurthe-et-Moselle : (36) Tronville, (46) } \\
\text { Bouxières-sous-Froidmont, (56) Belleau, (57) } \\
\text { Port-sur-Seille; } \\
\text { Meuse : (24) Rupt-en-Woëvre, (25) } \\
\text { Ranzières, (27) Seigneulles. }\end{array}$ \\
\hline - ALF & $\begin{array}{l}\text { Meurthe-et-Moselle : (170) Moncel-sur-Seille } \\
=\text { hót }(\text { vs. hótõo) } \\
\text { Jaulny },(171) \text { Mailly = finīiss, }(163)\end{array}$ \\
\hline - Adam (1881) : & $\begin{array}{l}\text { Meurthe-et-Moselle : Bouillonville, Mousson, } \\
\text { Thezey, Landremont, Moivrons, Battigny. } \\
\text { Ex. : } \\
\text { Moivrons, je minge, te minge, i minge, je } \\
\text { mingeans, vos minjoês, i mingent. } \\
\text { Je voi, te voi, i voi, je voyans, vos voyoês, i } \\
\text { voyent, } \\
\text { Landremont, je handele (je balaie), te } \\
\text { handele, i handele, je handelans, ve } \\
\text { hendeloôus i handelent, } \\
\text { Je mat (je mets), te mat, i mat, je mattans, ve } \\
\text { mattoôus, i mattent. }\end{array}$ \\
\hline - Dosdat (1909) : & $\begin{array}{l}\text { Moselle : (Canton de Pange) šãt (sg), šãtã, } \\
\text { šãta: }{ }^{i}, \text { šãt (pl) }\end{array}$ \\
\hline - This (1887) : & 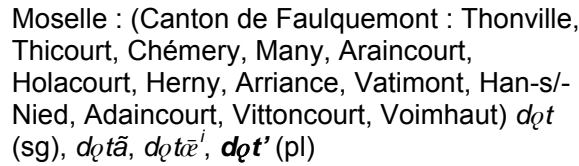 \\
\hline
\end{tabular}

Les localités concernées sont regroupées dans la zone d'extension maximale suivante :

\footnotetext{
${ }^{6}$ alld halten, au lieu de « finir ».

${ }^{7}$ «La troisième personne du pluriel se termine [E]n -ent, dans les communes de Bouillonville, Mousson, Thezey, Landremont, Moivrons, Battigny » (Adam, 1881:169). Tous les autres dialectes présentent ont/-ant ou -ot/-at, sauf Ventron et La Bresse.
} 


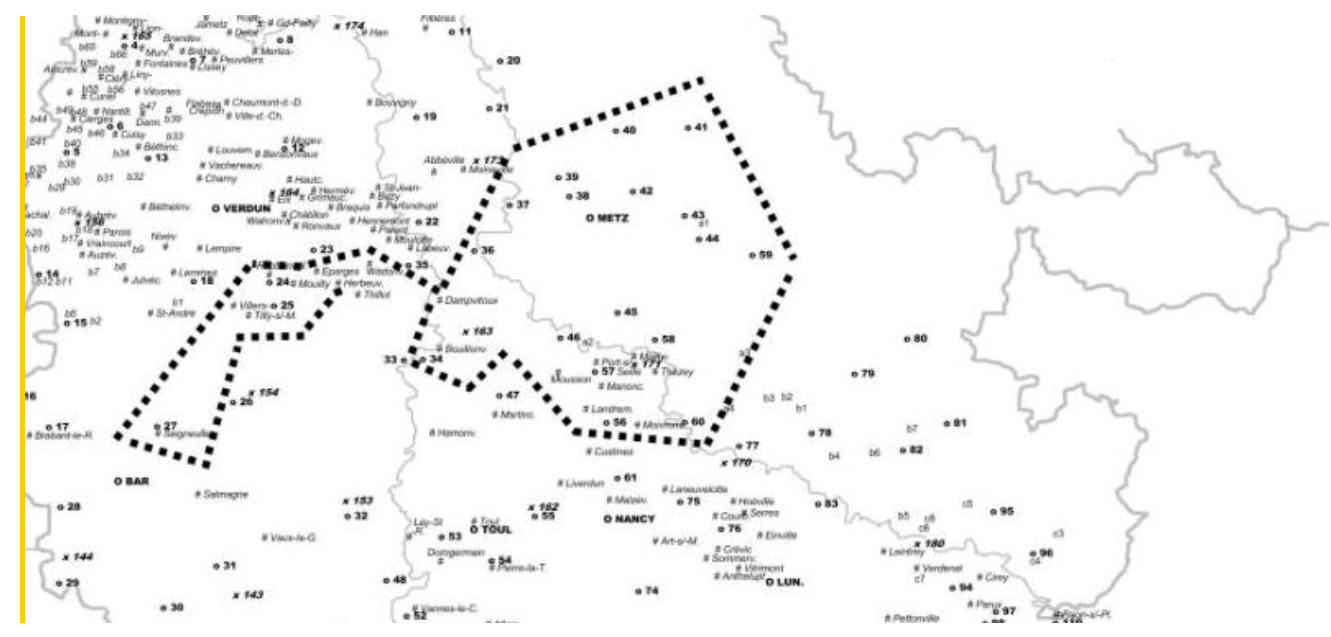

Figure 5 : Zone d'extension des formes verbales de P6 présent sans suffixe

Comme on le voit sur la carte, il se dessine une zone homogène autour de Metz, en Moselle, qui passe la frontière de Meurthe-et-Moselle dans le goulot étroit de ce département, et se rapproche, avec Bouillonville, de la frontière de la Meuse. En réalité, les localités de Meuse aux points 24 et 25, et plus au sud, 27, sont inscrites dans les mêmes latitudes que notre zone, et doivent donc manifestement y être intégrées, même si les points 154 de l'ALF et 26 et 33, peut-être 35, de l'ALLR, par exemple, n'y appartiennent pas et semblent couper la continuité de ce couloir qui s'arrête sur l'axe Verdun au nord et Bar-le-Duc au sud.

Il est essentiel de noter ici deux difficultés concernant le recueil des données en Meuse. En premier lieu, l'enquête d'Adam (1881) n'a pas publié les données du département de la Meuse, qui devraient pourtant être denses à la limite nord de notre zone (autour de Verdun). En second lieu, la guerre de 14-18 a été très destructrice dans cette région: les données dialectales modernes sont donc soit absentes, soit recueillies avec difficulté. Un des bons témoins du patois d'avant-guerre, Lavigne, nous permet d'ajouter quelques exemples hors de notre zone à Cumières-le-MortHomme, qui se trouve au nord-ouest de Verdun (Lavigne, 1929, d'après Méchin, 2008). P6 y est « normalement » marquée par une voyelle nasale :

Le père et la mère mingeont, qui criont, Is arrêtont lou ch'vau, îs racontront, ne pourtont poit de chapie, secquisont co, etc.

Il resterait encore à exploiter les volumes 5 (Meuse Nord) et 6 (Meuse Sud) des mémoires manuscrits envoyés par les correspondants de l'enquête de L. Adam et entreposés à la Bibliothèque Municipale de Nancy ${ }^{8}$. Grâce à l'aimable

\footnotetext{
${ }^{8}$ Il s'agit d'un ensemble de six volumes d'au moins 500 pages chacun, contenant parfois de véritables lexiques et esquisses grammaticales. L'essentiel est inédit.
} 
communication de J.-B. Picard, voici quelques données de P6 pour les communes de Wadonville, Les Éparges et Seigneulles :

Les Éparges : (être) y sont; (avoir) l'ont ; (corriger) y corrègent ; (se baigner) y se bognent ;

Wadonville: (être) y sont; (avoir) l'ont; (aimer) l'aïement; (se repentir) $i$ se repentent ;

Seigneulles : (avoir) l'on; (aimer) î l'ä̈me ; (se flatter) î s'flatte.

Les matériaux pour Seigneulles confirment les données de l'ALLR à un siècle de distance, puisqu'il s'agit du point 27, ceux de Wadonville et Les Éparges rapprochent de la frontière entre Meuse et Meurthe-et-Moselle : les graphies ent ou $e$ des verbes du premier groupe laissent peu de doutes sur l'absence de suffixation.

Une seule localité apparaît en dehors de notre zone : il s'agit de Battigny, tout à fait au sud de la Meurthe-et-Moselle, presque à la frontière des Vosges. Les conditions de recueil des données par L. Adam 9 , le fait que les données de Battigny sont simplement mentionnées sans être illustrées, et celui qu'elles ne sont pas soutenues dans le voisinage immédiat, nous engagent à ne pas nous fier à cette attestation isolée : il peut fort bien s'agir d'une erreur de transcription, ou d'une interférence avec le français standard.

On ne peut pas en dire autant de l'autre, et seule, zone, qui rayonne à partir d'un grand centre urbain, Metz, s'arrête à l'est à la frontière linguistique, mais se répand vers l'ouest au sud de l'axe Verdun-Metz, sans manifestement se confondre avec l'autre grand axe de communication lorrain, à savoir l'axe Bar-le-Duc-NancyLunéville. Il ne s'agit probablement pas d'une distribution aléatoire, mais d'une diffusion à partir de Metz d'une structuration paradigmatique calquée du français.

Du point de vue temporel, cette zone est manifestement déjà installée à la fin du $\mathrm{XIX}^{\mathrm{e}}$ siècle, et perdure au $\mathrm{XX}^{\mathrm{e}}$ siècle. Les données dialectales pour la période antérieure sont rares en dehors du pays messin.

Pour le XVIII ${ }^{\mathrm{e}}$ siècle, on peut toutefois prendre appui sur l'épopée messine de Chan Heurlin (Brondex \& Mory 1996 [1924]). La P6 y est régulièrement notée par la graphie francisante $<e n t>$, s'opposant ainsi à la $\mathrm{P} 4$ en $<a n s>$ :

P6 en -ent: Nas gens preignent austout «Nos gens prennent aussitôt» (102); Veignent près de Ginon "Viennent près de Ginon 》 (120); dijent mes caimérades « disent mes camarades》 (30); I r'watent « Ils regardent» (22); i sannent ses gayins «ils sentent ses fromages » (44); en cent endreus $i$ fornigent « en cent endroits ils fournissent » (70); I lo chuvent « Ils le suivent » (70), etc. ;

$\mathrm{P} 4$ ein -ans : $j$ ' allans « nous allons » $(30,54,66) ;$ Je n' volans mainget d'vos « Nous ne voulons manger de vous » (60); J'évans et fâre au moins « Nous avons à faire au

\footnotetext{
9 Enquête par correspondance, sans contre-enquête, auprès d'instituteurs, avec transcription orthographique manuscrite, variable, et francisante.
} 
moins » (66); Je n'nos mâlans jemâ « Nous ne nous mêlons jamais » (66) (voir aussi P4 à l'impératif).

De même la comédie La famille ridicule, du début du XVIII ${ }^{\mathrm{e}}$ siècle et représentant un dialecte mixte entre parler messin et parler du Sud-Est de Metz ${ }^{10}$, ne présente que des formes de P6 sans marque de personne. Nous donnons ci-dessous l'intégralité des exemples de P6, sauf formes redondantes, extraites du texte édité par Zéliqzon (1916) :

Tortos lés piates èfāres que s' pèssent dedans în m'néje « Toutes les petites affaires qui se passent [de]dans un ménage » (24)

Is sont zous cînq-ou chich, què bètent tojos l' pèvè «Ils sont [leur] cinq ou six, qui battent toujours le pavé » (27)

Que jâsent, et mè, j' n'oūs'reūs sol'mant ovri lè boche "Qui jasent, et moi, je n'oserais seulement ouvrir la bouche » (29)

J'antands dés jans que hoūyent au tau «J'entends des gens qui appellent dans la boutique » (34)

De guèchons qu'vègnent cheuz nos, is sont cînq que lè d'mandent « De garçons qui viennent chez nous, ils sont cinq qui la demandent » $(42)^{11}$

Que l' inment è lè folè-ye "Qui l'aiment à la folie » (43)

Mas po lés pracurous, is guingnent de mout bones somes « Mais pour les avocats, ils gagnent de très belles sommes » (45)

$S$ 'is veuyent que j' fèsse por zous «S'ils veulent que je fasse (quelque chose) pour eux » (46)

Qu'an pèyent lés-intèrèts " Qui en payent les intérêts. » (49)

Is $m$ ' èpèlent loup cerviè « Ils m'appellent loup cervier » (55)

E't pus is r'vègnent sotit « Et puis ils reviennent à la maison » (79)

Is malträtèyent eune fome èt sovant lè veuyent bète «Ils maltraitent une femme et souvent la veulent battre» (79)

Que nos sèrvent pus de ryin "Qui nous servent plus de rien » (82)

que $m$ ' roütent d'în grand soci « qui m'ôtent [d']un grand souci » (90)

Is sont an haut que pālent d'èfāres « Ils sont en haut qui parlent d'affaires » (90)

Nas janes jans n'en peuyent pus, is meurent s'on n' lés mèrèye « Nos jeunes gens n'en peuvent plus, ils meurent si on ne les marie » (107)

Que s' ambrèssent èt que s' bajent «Qui s'embrassent et qui se baisent » (107)

Is vègnent $c a$ « Ils viennent encore » (110)

Les verbes qui seraient en français standard à base unique, type passer, gagner, jaser, ôter, etc., à base multiple, en « $\mathrm{L}$ » comme appeler, mourir ou en « U » comme battre, servir, aussi bien que les verbes irréguliers comme venir ou vouloir, présentent tous la même caractéristique d'une P6 marquée uniquement par une base à finale consonantique.

${ }^{10}$ «Das Stück ist in der Mundart geschrieben, wie sie in Metz und den Dörfern seiner nächsten Umgebung gebräuchlich ist oder vielmehr war. Wir haben es mit einem Mischdialekt zu tun, der Eigenheiten des im Nordosten von Metz gesprochenen eigentlichen Patois messin enthält und der Mundart, die im Seilletal südöstlich der Stadt heimisch ist. » (Zéliqzon éd., 1916 : 12). Signalons que L. Zéliqzon, bon connaisseur des parlers de Moselle, a entièrement retranscrit le texte, à l'origine dans une orthographe tout à fait fantaisiste. Nous sommes confiant qu'il aurait discuté en note des variantes orthographiques surprenantes (cf. note 12 et infra).

${ }^{11}$ Rime avec eune bande « une bande ». 
Cette association de P6 avec une finale graphique muette $<e n t>$ dans les textes modernes trouve une confirmation dans les textes du Moyen Âge. Par exemple, le Psaultier de Metz, texte du XIV ${ }^{\mathrm{e}}$ siècle, montre clairement un paradigme aaadea pour les verbes du premier groupe, ce qu'indiquent les variantes relevées par Apfelstedt (1881 : lv-lvi, voir aussi Bonnardot 1884) :

Tableau 7 : Paradigme verbal du Psaultier de Metz
\begin{tabular}{|l|l|l|}
\hline P1 & $<-\underline{e}>$ & annunce \\
\hline P2 & $<-\underline{e} s>,<-e>$ & $\begin{array}{l}\text { enlumines ; affaice, glorifie, montre, } \\
\text { refuse, salue }\end{array}$ \\
\hline P3 & $<-\underline{e}>,<-e t>,(<-e n t>)$ & $\begin{array}{l}\text { assemblet, cesset, habitet ; appellent, } \\
\text { fient }\end{array}$ \\
\hline P4 & $<-\underline{\text { on}} s>,(<-o n>)$ & \\
\hline P5 & $<-\underline{e}-\mathbf{z}>,<-e i s>,(<-e i>)$ & $\begin{array}{l}\text { habiteis, Imp. chanteis, distribueis ; } \\
\text { appellei }\end{array}$ \\
\hline P6 & $<-\underline{e} n t>,<-e>$ & $\begin{array}{l}\text { fient, racontent ; cesse, monte, } \\
\text { ordene, pance, parle }\end{array}$ \\
\hline
\end{tabular}

C'est une graphie <ent> que l'on trouve également pour des verbes d'autres types, par exemple vouloir ${ }^{12}$ : de ceulz qui de ti ne welent approchieir et Soient mis darrieir et aient honte. qui mal me welent.

Il semblerait donc que la perte d'un marquage personnel pour P6, et ce faisant, la réorganisation du paradigme lorrain du présent à Metz et aux alentours, se soient établies dès le Moyen Âge et probablement sous l'influence du français écrit.

La qualité de foyer de diffusion culturelle de Metz a été soulignée entre autres par F. Bonnardot ou L. Zéliqzon :

Metz doit à sa situation géographique d'avoir toujours été un foyer actif de culture et d'expansion de la langue française : traduction des livres saints, chansons de geste, poèmes historiques et romans d'aventures, atours officiels et chartes d'intérêt privée, relations de voyages et de pèlerinages, mystères dramatiques, chroniques et légendes, contes et nouvelles, mémoires, chansons tant en français pur que dans le dialecte local, toutes les branches de la littérature ont été cultivées avec ardeur durant une longue période qui correspond à celle de l'indépendance de la république messine. (Bonnardot, $1891: 331$ )

Metz a été une ville active dans la diffusion des variétés écrites et orales aussi bien du français que des dialectes. Le fait qu'on trouve précisément dans cette région des formes qui ne correspondent pas au type normal des dialectes lorrains, mais au contraire des formes similaires à celles du français doit être mis au compte

${ }^{12}$ Cf. aussi : «In der 2. schwachen Conjug. sind im Sg. die Endconsonanten verstummt: descen (1.) 138,8; entens (3.) pr. 4, 35; die 1. u. 2. Ps. Pl. sind nicht belegt, din $<$ sic $>3$. zeigt graphisch immer ent. » (ibid.) 
d'une influence à partir du français standard, dans une situation de type « diaglossique » (Auer, 2005). Inversement, des formes éloignées du standard, telles qu'une première personne vocalique de type $j$ 'es pour « je suis » ont pu jouxter Metz sans y entrer (cf. Duval, 2010).

La période à laquelle des formes françaises se sont introduites dans les dialectes ne pourra sans doute pas être déterminée avec précision, ne serait-ce qu'en raison de la pauvreté des données dialectales jusqu'au XIX ${ }^{\mathrm{e}}$ siècle, et de leur faible fiabilité.

Parmi ces données difficiles à exploiter, trois textes du pays messin retiennent toutefois notre attention. Tout d'abord, la Charte des Chaiviers du $15^{\mathrm{e}}$ siècle, éditée par Bonnardot (1891), mais de lecture difficile ${ }^{13}$, présente deux formes en $<$-ont $>$, manifestement du premier groupe :

col lou maiste sai cordont $\mid$ a $\boldsymbol{\tau}$ tornont parlou cran et parlaicor de toucos (version B) « [...] avec le maître s'accordent et <attornent> [...] (??)»

Dans La grosse enwaraye $\left(15^{\mathrm{e}}-16^{\mathrm{e}}\right)$, comédie grivoise qui montre des traits dialectaux du Pays-Haut (zone P chez L. Zéliqzon: Amanvillers, Avril, etc.), on relève une forme en $<$ on $>$ pour « vouloir » $\mathrm{P} 6$, mais des formes sans suffixe pour les verbes de premier groupe « pisser » et « chier » :

$Y$ volon tra vvaignié dedgen « Ils veulent trop gagner d'argent » Au moin se lé bacelle y peuche / Se seret on klés aute chée "Au moins si les filles y pissent, Ce sera où que les autres chient »

Il est intéressant de voir ici une alternance entre deux types de marquage. Or, dans un texte beaucoup plus récent, l'Éloge historique du Sieur Vernier (publiée en 1844, mais écrite probablement à la fin du XVIII ${ }^{\mathrm{e}}$ siècle $^{14}$ ), L. Zéliqzon (1928) avait aussi noté une alternance entre finale en -on et finale en -en. Nous citons ici le commentaire qu'il consacre au vers $62^{15}$ :

Y N'RANJEONS JEMA, ils ne rangent jamais. Ajouter : y n'manquont jema (262), y fournissons (353), à côté de: les gens qu'aiment (343), ils rendent (351). La 3e personne du pluriel du présent de l'indicatif se termine tantôt en -ons $(t)$, tantôt en -ens $(t)$. Le patois ne connait que la forme en ent. La terminaison en -ons $(t)$ ne peut appartenir qu'au français populaire ; cependant, je ne l'ai jamais entendue, ni à Metz, ni à la campagne. Il est probable que l'abbé Georgin, en l'adoptant, a voulu donner à son poème une couleur soi-disant plus dialectale; ce peut être aussi du patois « littéraire », les paysans de Molière, etc., parlent ainsi. (Zéliqzon, $1928: 64$, note 62)

On voit que L. Zéliqzon suppose une erreur, consciente ou non, de la part de l'auteur, et ne reconnaît comme vraisemblablement dialectales que les graphies

\footnotetext{
${ }^{13} \mathrm{~F}$. Bonnardot lui-même ne se hasarde pas à en donner une traduction.

${ }^{14}$ Texte, assez mélangé de français, composé par l'abbé Chrétien-Nicolas Georgin (1753-1794) né à Metz.

${ }^{15}$ En remerciant Jean Richard de nous avoir prêté cet article de L. Zéliqzon auquel nous n'avions pu accéder jusqu'à la rédaction finale de ce texte, et qui a beaucoup alimenté notre réflexion.
} 
muettes en $\langle e n\rangle$, non suffixées. Si le texte est effectivement d'une transcription peu fiable, il est fort possible que L. Zéliqzon, excellent connaisseur des dialectes mosellans, n'ait pas vu la singularité des formes non-suffixées de P6 en Lorraine : en effet, ce phénomène occupe une large part de l'espace dialectal mosellan. Or, d'un point de vue régional, ce sont les formes notées $<e n>$ qui sont surprenantes, alors que les finales en $<$ on $>$ paraissent tout à fait normales.

$\mathrm{Si}$ seules trois formes rejetées par L. Zéliqzon sont en effet en $<$ on> $(Y$ $n$ 'rangeons (62), in manquont « ils ne manquent» (262), y fournissons (353) $)^{16}$, il n'y en que six en $\langle e(n)>$ (Les gens veuillent «veulent» (69), Et y n'en trove «ils n'en trouvent » (80), il sève «ils savent» (211), qu'aiment (243), Ils rendent (351), deuve «doivent» [les banques] (475)), et les diverses occurrences d'une même forme ne varient pas (cf. veuillent aux vers $69,74,75,480$ ).

Il est donc envisageable qu'une période ait été marquée par une alternance de marquage selon les verbes. Les causes de cette alternance resteraient encore à définir. On peut remarquer que toutes celles sans suffixe se terminent toujours par une consonne sonore, mais ce trait n'est pas distinctif par rapport à trove «trouvent». Les verbes impliqués appartiennent par ailleurs à des classes morphologiques distinctes. Il est certainement plus plausible de penser que le texte de l'abbé Georgin montre simplement une situation instable de variation entre deux paradigmes : aaadef ${ }^{17}$ vs paradigme aaadea, dont on sait que ce dernier finira par s'imposer autour de Metz. Si l'on ajoute à cela l'argument des paradigmes aberrants faisant apparaître un - $n$ final pour les formes de type finissent en bordure sud-est de notre zone, cela nous conforte dans l'idée que la répartition des formes nonsuffixées de P6 autour d'un grand centre urbain diffuseur de formes françaises n'a pas évolué de façon indépendante pour aboutir par miracle au même système que celui de l'ouest d'oïl. Les formes en <on> ne relèvent certainement pas d'un hypothétique français populaire que L. Zéliqzon avoue n'avoir jamais entendu, mais au contraire de dialectes, et c'est bien ces formes qui ont dû disparaître de la région messine sous la pression des variétés écrites et orales d'un français tout à fait standard, qui a probablement influencé en premier lieu les variétés dialectales hautes de messin, pour s'établir ensuite en périphérie avec éventuellement quelques arrangements morphologiques rendant les paradigmes locaux variables ou aberrants.

Du point de vue systémique, on peut rappeler l'hypothèse que le présent dans les parlers romans est très fortement contraint par sa solidarité avec l'impératif, ce qui l'empêche d'aboutir en Lorraine à une conjugaison biflexionnelle opposant simplement singulier et pluriel, comme c'est le cas par ailleurs (la distinction entre personnes étant assurée par les clitiques sujets). P4 et P5 devant être différenciés, P6 reste donc libre de s'aligner sur une forme interne au paradigme, ou bien de se calquer sur une forme empruntée.

\footnotetext{
${ }^{16}$ A côté toutefois des formes attendues vont, sont, ont, font identiques au français standard. Celles-ci ont d'ailleurs pu contribuer à la conservation de <-on> comme marque de P6 pour les autres verbes (cf. aussi les futurs veuront « viendront » (254), ils to conduront « ils te conduiront» (437), qui vinront et queront « qui viendront et qui auront » (521)).

${ }^{17}$ La première personne y étant normalement en an $(s)$ : porchuvans « poursuivons », vollant « voulons », allans «allons", évan "avons", travaillant «travaillons", mais une fois pouvons, et plusieurs occurrences de allons employé comme connecteur de discours.
} 


\section{Références}

[alf] Gillieron J. \& Edmont, E. (1903-1910), Atlas linguistique de la France, Paris : Champion.

[ALLR] LANHeR J., LitAize A. \& RichaRd J. (1979-1988) Atlas linguistique et ethnographique de la Lorraine romane. Tome I (1979), Tome II (1981), Tome III (1985), Tome IV (1988), Breitenau, CNRS.

ADAM L. (1881), Les patois lorrains, Nancy, Grosjean-Maupin.

APFELSTEDT F. (éd.) (1881), Lothringischer Psalter (Bibl. Mazarine, no. 798), altfranzösische Übersetzung des XIV. Jahrhunderts, mit einer grammatischen Einleitung, enthaltend die Grundzüge der Grammatik des altlothringischen Dialects, und einem Glossar..., Heilbronn : Gebr. Henninger.

AUER P. (2005), « Europe's sociolinguistic unity, or: A typology of European dialect/standard constellations ", in N. Delbecque, J. van der Auwera \& D. Geeraerts (éds), Perspectives on variation: Sociolinguistic, historical, comparative, Berlin / New York : de Gruyter, 742.

BABIN J. (1954), Les parlers de l'Argonne, Paris : Klincksieck.

BLoch O. (1917), Atlas linguistique des Vosges méridionales, Paris : Champion.

Bonnardot F. (1884), Le Psautier de Metz: Texte du XIV siècle, édition critique publiée d'après quatre manuscrits, Tome premier: Texte intégral, Paris : F. Vieweg.

Bonnardot F. (1891), «Trois textes en patois de Metz: Charte des Chaiviers, La grosse anwaraye, Une fiauve recreative (XV $-\mathrm{XVII}^{\mathrm{e}}$ siècles) », in Études romanes dédiées à Gaston Paris le 29 Décembre 1890 (25 anniversaire de son doctorat ès lettres) par ses élèves français et ses élèves étrangers des pays de langue française, Paris : E. Bouillon, 331-405.

Borodina M. A. (1966), Problemy lingvisticheskoj geografii, Moskva : Nauka.

Brod R. (1912), Die Mundart der Kantone Château-Salins und Vic in Lothringen, Halle a. S. : Ehrhardt Karras.

BrondeX A. \& Mory D. (1996 [1924]), Chan heurlin ou les fiançailles de Fanchon, Metz: Serpenoise.

Dosdat F. (1909), «Die Mundart des Kantons Pange (Kreis Metz in Lothringen)», Zeitschrift für romanische Philologie 33, 187-225, 257-276.

Duval M. (2009), «L'homonymie des marques personnelles à l'est d'oïl (1) : l'indicatif présent ", in H.-R. Nüesch, H.-R. (éd.), Galloromanica et Romanica. Mélanges de linguistique offerts à Jakob Wüest, Tübingen, Narr Francke Attempto, 71-86.

Duval M. (2010), "J'es, tu es, il est: un problème de dialectologie lorraine », Revue de Linguistique Romane 74, 341-414.

HoRNING A. (1887), «Die ostfranzösischen Grenzdialekte zwischen Metz und Belfort», Französische Studien 5:4 , 429-550. [n'est pas cité dans le corps de l'article]

Mechin C. (2008), Louis Lavigne et Cumières, village mort pour la France, suivi de deux textes de Louis Lavigne : La vie de Sébastien Anclin, aveugle (1874-1938) et Sur le pouce [Petites histoires meusiennes en patois de Cumières], Régneville-sur-Meuse, Les Dossiers Documentaires Meusiens.

MEYER-LÜBKE W. (1895), Grammaire des langues romanes, Vol. 2 : Morphologie. Traduit par Auguste Doutrepont et Georges Doutrepont, Paris : H. Welter.

THIS C. (1887), Die Mundart der französischen Ortschaften des Kantons Falkenberg (Kreis Bolchen in Lothringen), Strassburg: J. H. Ed. Heitz. 
ZÉLIQZON L. (1889), Lothringische Mundarten, Metz: G. Scriba.

ZeliQzon L. (1922), Dictionnaire des patois romans de la Moselle, Strasbourg: Librairie Istra.

ZELIQZON L. (1928), «Éloge historique du sieur Vernier», Annuaire de la Société d'histoire et d'archéologie de la Lorraine 37, 35-68.

ZELIQZON L. (éd.) (1916), La famille ridicule, comédie messine en vers patois, Metz : Verlag der Gesellschaft für lothringische Geschichte und Altertumskunde.

ZELIQZON L. \& THIRIOT, G. (éds) (1912), Textes patois recueillis en Lorraine, Metz : Verlag der Gesellschaft für lothringische Geschichte und Altertumskunde. [je ne l'ai pas retrouvé dans le corps de l'article] 